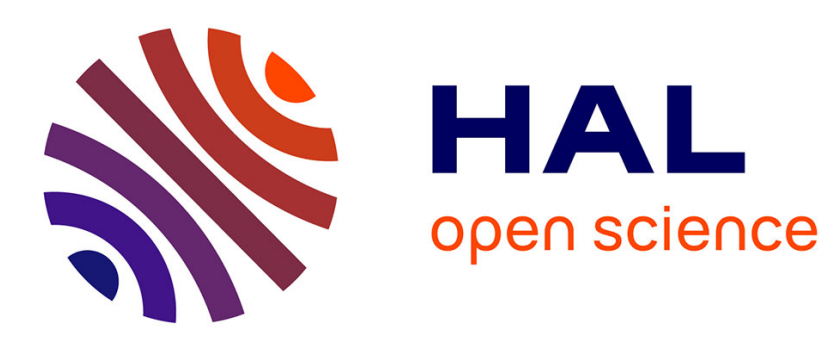

\title{
A Simple hypothesis for the spin glass phase of the pnfinite-ranged SK model
}

\author{
G. Parisi, G. Toulouse
}

\section{To cite this version:}

G. Parisi, G. Toulouse. A Simple hypothesis for the spin glass phase of the pnfinite-ranged SK model. Journal de Physique Lettres, 1980, 41 (15), pp.361-364. 10.1051/jphyslet:019800041015036100 . jpa00231798

\section{HAL Id: jpa-00231798 https://hal.science/jpa-00231798}

Submitted on 1 Jan 1980

HAL is a multi-disciplinary open access archive for the deposit and dissemination of scientific research documents, whether they are published or not. The documents may come from teaching and research institutions in France or abroad, or from public or private research centers.
L'archive ouverte pluridisciplinaire HAL, est destinée au dépôt et à la diffusion de documents scientifiques de niveau recherche, publiés ou non, émanant des établissements d'enseignement et de recherche français ou étrangers, des laboratoires publics ou privés. 


\title{
A Simple Hypothesis for the Spin Glass Phase of the Infinite-Ranged SK model
}

\author{
G. Parisi \\ INFN, Frascati, Roma, Italia \\ and G. Toulouse \\ Laboratoire de Physique de l'Ecole Normale Supérieure, 24, rue Lhomond, 75231 Paris 05, France
}

(Reçu le 29 avril 1980, accepté le 11 juin 1980)

\begin{abstract}
Résumé. - On montre qu'une hypothèse simple, dans le cadre de la théorie de champ moyen de la phase verre de spin, reproduit maints résultats souhaités. En outre, cette hypothèse mène à des prédictions (sauts faibles pour la chaleur spécifique et pour la susceptibilité magnétique, en champ fini) qui fournissent des tests cruciaux pour sa validité.
\end{abstract}

Abstract. - A simple Hypothesis for the mean field theory of a spin glass phase is shown to reproduce many desired features. Besides, it leads to predictions (weak jumps for the specific heat and the magnetic susceptibility, in finite field) which provide crucial tests for its validity.

1. Introduction. - The infinite-ranged model of a spin glass which was introduced by $\mathrm{D}$. Sherrington and S. Kirkpatrick (SK) [1] is expected to have a solution of the mean field type. In an $(H, T)$ phase diagram, where $H$ is a uniform magnetic field and $T$ is the temperature, there appears to exist a dividing line [2] where the high temperature phase becomes unstable. What exactly occurs in the low temperature spin glass phase remains somewhat problematic, with hints coming from Monte-Carlo simulations and replica symmetry breaking schemes, and also from the related random energy model of B. Derrida [3].

It is shown here that a drastically simple extrapolation procedure, projecting physical properties from the instability line on to the spin glass phase, reproduces many of the expected features, including various details predicted by the most recent replica symmetry breaking scheme [4]. The crucial prediction of this projection Hypothesis is the existence of (weak) jumps for the specific heat and the magnetic susceptibility across the instability line.

2. The projection Hypothesis for the spin glass phase. - Suppose that, within the spin glass phase of the SK model,

$$
\begin{aligned}
& S(T, H)=S(T), \\
& M(T, H)=M(H), \\
& q(T, H)=q(T),
\end{aligned}
$$

where $S$ is the entropy, $M$ the magnetization and $q$, the Edwards-Anderson order parameter, is defined as $\bar{m}^{2}$, where $m$ is the magnetization in each of the possible realizations of the set of random couplings and the bar denotes the average over the different realizations. Formulae (1) and (2) are not independent, because of the Maxwell relation :

$$
\frac{\partial S}{\partial H}=\frac{\partial M}{\partial T} .
$$

Armed with such formulae, one is able to make detailed predictions for the properties of the spin glass phase, starting from the instability line, where everything can be calculated without replica symmetry breaking. In pratice, analytic expansions can be performed around the two ends of the instability line : $\left(H \simeq 0, T \simeq T_{\mathrm{c}}\right)$ and $(H \simeq \infty, T \simeq 0)$, and numerical estimates in between.

3. Physical quantities on the instability line. - The position of the instability line [2] is defined by :

$$
T^{2}=\frac{1}{\sqrt{2 \pi}} \int_{-\infty}^{+\infty} \mathrm{d} z \exp \left(\frac{z^{2}}{2}\right) \operatorname{sech}^{4}\left(\frac{z \sqrt{q}}{T}+\frac{H}{T}\right)
$$

where, for notation simplicity, the usual variance parameter $J$ for the bond distribution has been taken 
equal to unity, as well as Boltzmann constant, so that $T_{\mathrm{c}}(H=0)=1$.

In the vicinity of the transition point in zero field, the line equation may be written :

$$
H^{2} \simeq \frac{4}{3} \tau^{3}\left(1+\frac{\tau}{3}\right)
$$

where $\tau=T_{\mathrm{c}}-T=1-T$. The other end of the line is given by :

$$
\begin{aligned}
T \simeq \frac{2}{3} \sqrt{\frac{2}{\pi}} & \exp \left(-\frac{H^{2}}{2}\right) \times \\
\times & {\left[1+T^{2}\left(H^{2}-1\right)\left(\frac{\pi^{2}}{24}-1\right)\right] . }
\end{aligned}
$$

The first terms in both expansions have already been computed by de Almeida-Thouless [2].

Starting from $(H=0, T=1)$ along this instability line, one obtains the following expansions :

$$
\begin{aligned}
& q=\tau+\tau^{2}-\tau^{3}+\mathrm{O}\left(\tau^{4}\right), \\
& S=\log 2-\frac{1}{4}-\frac{\tau}{2}-\frac{\tau^{2}}{4}+\frac{\tau^{3}}{6}-\frac{\tau^{4}}{24}+\mathrm{O}\left(\tau^{5}\right), \\
& M \simeq H-\left(\frac{3}{4}\right)^{2 / 3} H^{7 / 3}+\frac{2}{3} H^{3} .
\end{aligned}
$$

Starting from the other end, one gets :

$$
q \simeq 1-\frac{3}{2} T^{2}-\frac{3}{8} T^{4}\left(H^{2}-1\right),
$$

where, according to (7), $H^{2} \simeq-2 \log \left(\frac{3}{2} \sqrt{\frac{\pi}{2}} T\right)$,

$S \simeq \frac{2 \pi^{2}-9}{16} T^{2}$

$M \simeq M_{\mathrm{SK}}^{0}+\frac{\left(18-\pi^{2}\right)}{27 \pi} \sqrt{\frac{2}{\pi}} H \exp \left(-\frac{3}{2} H^{2}\right)$,

where $M_{\mathrm{SK}}^{0}(H)$ is the zero temperature magnetization calculated without replica symmetry breaking :

$$
M_{\mathrm{SK}}^{0}(H)=\frac{1}{\sqrt{2 \pi}} \int_{-H / V \bar{q}}^{+H / \sqrt{q}} \mathrm{~d} z \exp \left(-\frac{z^{2}}{2}\right) .
$$

4. Predictions. - From (3) and (8), the prediction for the parameter $q$, in the vicinity of $T_{\mathrm{c}}$, is in agreement with the prediction of A. Bray and M. Moore [5]. From (3) and (11), one predicts that $q$ behaves at low temperatures like

$$
q \simeq 1-\alpha T^{2}
$$

with a value of $\alpha=\frac{3}{2}$, which is to be compared with the TAP [6] prediction, $\alpha=2 \sqrt{\log 2} \simeq 1.665$, and the Bray-Moore [5] prediction, $\alpha \simeq 1.810$.

If we make the hypothesis that $q_{4}=\overline{m^{4}}$ is also independent of the magnetic field, we find directly from (5) :

$$
q_{4}-2 q+1=T^{2}
$$

which is also a prediction of Bray and Moore [5].

From (1) and (9), one obtains a prediction for the entropy, in the vicinity of $T_{c}$. As a consequence, one gets :

$$
\delta F=\frac{2}{45} \tau^{5}+\mathrm{O}\left(\tau^{6}\right)
$$

where $\delta F$ is the free energy measured relative to the SK solution (without replica symmetry breaking). This agrees with the predictions of Parisi [4]. From (1) and (12), the low temperature entropy is predicted to start from zero with a quadratic temperature term, the coefficient of which :

$$
\frac{2 \pi^{2}-9}{16} \simeq 0.671
$$

is to be compared with the TAP [6] : 0.770 and BrayMoore [5] : 0.765 predictions.

From (2), (10) and (13), one predicts that the magnetic susceptibility behaves as :

$$
\chi(H) \simeq 1-\frac{7}{3}\left(\frac{3}{4}\right)^{2 / 3} H^{4 / 3}+2 H^{2},
$$

for $H$ small, and

$$
\begin{aligned}
\chi(H) \simeq & \sqrt{\frac{2}{\pi}} \exp \left(-\frac{H^{2}}{2}\right) \times \\
& \times\left[1+\frac{\left(18-\pi^{2}\right)}{27 \pi}\left(1-3 H^{2}\right) \mathrm{e}^{-H^{2}}\right],
\end{aligned}
$$

for $H$ large but still smaller than $H_{\mathrm{c}}(T)$, the critical field corresponding to the instability line. Formula (18) is in qualitative agreement with the predictions of Parisi [4].

It is instructive to sketch the predictions of (18) and (19) for the zero temperature susceptibility, and to make a comparison with the SK value (calculated without replica symmetry breaking) $\chi_{\mathrm{SK}}^{0}(H)$ :

$$
\chi_{\mathrm{SK}}^{0}(H)=\sqrt{\frac{2}{\pi}} \exp \left(-\frac{H^{2}}{2}\right) .
$$

This is done in figure 1 . Obviously, since the area under each curve is equal to unity, and since the difference in the ground state energies in zero field is given by :

$$
\delta E_{0}=-\int_{0}^{\infty} H \delta \chi(H) \mathrm{d} H
$$

such behaviour for the susceptibility is in agreement with a ground state energy higher than the SK value. 


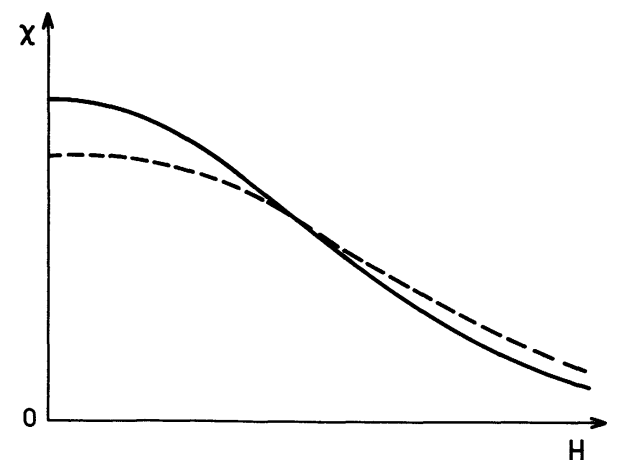

Fig. 1. - The full line is the prediction for the zero temperature susceptibility. The dashed line is the SK result :

$$
\chi_{\mathrm{SK}}^{0}=\sqrt{\frac{2}{\pi}} \cdot \exp \left(-\frac{H^{2}}{2}\right) .
$$

A numerical estimate for the ground state energy $E_{0}$ may be computed, but this has not yet been done.

5. Discussion. - The reason why this projection procedure gives sound results can be understood in simple terms. Consider the entropy $S_{\mathrm{SK}}(T, H)$, calculated without replica symmetry breaking, as a function of $H$ (Fig. 2). For $T>T_{\mathrm{c}}$, the entropy is a monoto-

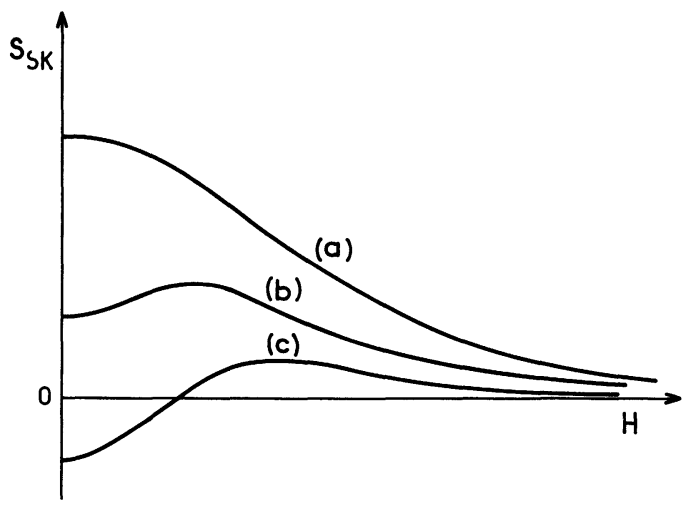

Fig. 2. - The entropy, as calculated without replica symmetry breaking ; (a) at high temperature, $T>T_{\mathrm{c}}$; (b) and (c) at increasingly lower temperature, $T<T_{\mathrm{c}}$

nously decreasing function of $H$. For $T<T_{\mathrm{c}}$, the entropy $S_{\mathrm{SK}}$ presents a maximum for a finite value of $H$ (in a way which is reminiscent of antiferromagnetic behaviour). The locus of this maximum of $M_{\mathrm{SK}}(T)$ according to (4), is given by :

$$
H^{2} \simeq \frac{4}{3} \tau^{3}(1-2 \tau),
$$

for $H$ small, and

$$
T \simeq \frac{6}{\pi^{2}} \sqrt{\frac{2}{\pi}} \exp \left(-\frac{H^{2}}{2}\right),
$$

for $T$ small.

This locus is therefore very close to the instability line. Now the true entropy is expected to depart very mildly from the SK entropy below the instability (remember that, on the zero-field axis,

$$
\left.\delta S=S-S_{\mathrm{SK}}=\mathrm{O}\left(\tau^{4}\right)\right) .
$$

Since the entropy is close to stationary on the instability line and has to be stationary on the zero-field axis, it must be a very slowly varying function of $H$ in between. Indeed it has been argued, in the replica broken symmetry approach of [4], that :

$$
\left.\frac{\hat{\partial} \chi}{\hat{c} T}\right|_{H=0}=\left.\frac{\hat{c}^{2} S}{\partial H^{2}}\right|_{H=0}=0 .
$$

However, this projection Hypothesis also predicts jumps for the specific heat $\Delta C$ and for the magnetic susceptibility $\Delta \chi$, across the instability line. Thus, for $H \simeq 0$,

$\Delta \chi=\chi($ low temperature side $)-$

$$
-\chi \text { (high temperature side) }
$$

$$
\simeq \frac{5}{18} H^{2},
$$

whereas, for $T \simeq 0$,

$$
\Delta C \simeq \frac{\pi^{2}-9}{8} T^{2} .
$$

The values of the jumps, at one point on the instability line $H_{\mathrm{c}}(T)$, are related by equations involving the slope of the line :

$$
\frac{\Delta C}{T}=-\Delta A \cdot \frac{\mathrm{d} H_{\mathrm{c}}}{\mathrm{d} T}=\Delta \chi \cdot\left(\frac{\mathrm{d} H_{\mathrm{c}}}{\mathrm{d} T}\right)^{2},
$$

where $A=\partial S / \partial H=\partial M / \partial T$. These equations are for the instability line, predicted here to be second order, what the Clausius-Clapeyron equation is for a first order transition line.

Now, at the two ends of the instability line, one finds :

$$
\Delta A>0,
$$

and therefore, since $\mathrm{d} H_{\mathrm{c}} / \mathrm{d} T<0, \Delta C$ and $\Delta \chi$ are positive (see Fig. 3 for a sketch of the magnetic susceptibility in low field).

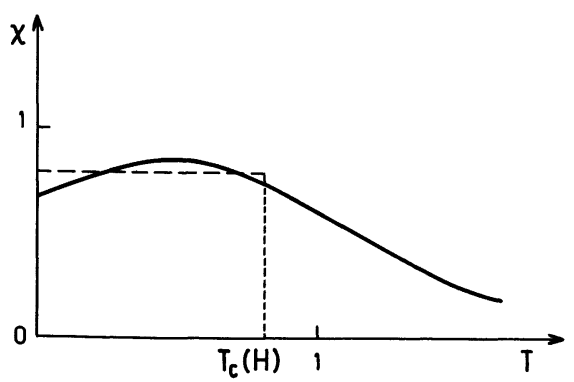

Fig. 3. - The full line represents the magnetic susceptibility $\chi_{\text {sk }}(T, H)$, calculated without replica symmetry breaking, in a small applied field $H$. The horizontal dashed segment is the prediction of the projection Hypothesis, in the spin glass phase. All three figures are free hand drawings. 
In the intermediate region, rough numerical calculation seem to indicate that $\Delta A$ remains very small. The predicted jumps would therefore be tiny.

Thus the projection Hypothesis predicts that the instability line is a second order line (in the Ehrenfest sense), terminating at a third order point on the zerofield axis. Note that it cannot be a third order line, with cusps for $\chi$ and $C$, because one would have :

$$
\frac{\partial}{\partial T}\left(\frac{\Delta C}{T}\right)=\frac{\partial}{\partial T}(\Delta \chi) \cdot\left(\frac{\mathrm{d} H_{\mathrm{c}}}{\mathrm{d} T}\right)^{2},
$$

i.e. a relation which has an unacceptable limit on the zero-field axis. However, it is also possible that the correct solution does not have jumps, as in the approaches of $[4]$ and $[5]$. which at tually would rather suggest that the instability line is a line of fourth order transition.

As a final illustration of the contents of the projection Hypothesis, we give here an exact thermodynamic expression, relating various quantities of interest :

$$
\begin{aligned}
& E_{0}+\int_{0}^{T} C\left(T^{\prime}\right) \mathrm{d} T^{\prime}=-\frac{\left(1-q^{2}\left(T, H_{\mathrm{c}}\right)\right)}{2 T}- \\
& -\int_{0}^{H_{\mathrm{c}}} \chi\left(T, H^{\prime}\right) H^{\prime} \mathrm{d} H^{\prime}-T\left[S\left(T, H_{\mathrm{c}}\right)-S(T, 0)\right],
\end{aligned}
$$

$T<T_{\mathrm{c}}$, and the reduced form that it takes, within the present set of hypotheses :

$$
\begin{aligned}
& E_{0}+\int_{0}^{T} C\left(T^{\prime}\right) \mathrm{d} T^{\prime}= \\
& \quad=-\frac{\left(1-q^{2}(T)\right)}{2 T}-\int_{0}^{H_{\mathrm{c}}} \chi\left(H^{\prime}\right) H^{\prime} \mathrm{d} H^{\prime} .
\end{aligned}
$$

6. Conclusion. - A desired feature for a mean field theory is simplicity, in order to allow the treatment of fluctuations in a later state. The projection Hypothesis presented here is an attempt at simplicity. But is simplicity, in the mean field theory of spin glasses, compatible with truth, which is another desirable feature ? The most direct check passes by a more accurate determination, numerical or otherwise, of the nature of the singularities on the instability line.

Acknowledgments. - One of us (G. P.) is happy to thank the Universite Paris VI and the Laboratoire de Physique de l'Ecole Normale Supérieure for the warm hospitality during the preparation of this note.

\section{References}

[1] Sherrington, D., KirkPatrick, S., Phys. Rev. Lett. 32 (1975) 1792 ;

KiRKPatrick, S., Sherrington, D., Phys. Rev. B 17 (1978) 4384

[2] De Almeida, J. R. L., Thouless, D. J., J. Phys. A 11 (1978) 983.

[3] Derrida, B., to appear in Phys. Rep., Proc. of the Les Houches Institute (1980).
[4] PARISI, G., Phys. Lett. A 73 (1979) 203; Phys. Rev. Lett. 23 (1979) 1754 ; J. Phys. A 13 (1980) 1101 ; to appear in J. Phys. A 13 (1980); to appear in Philos. Mag.

[5] Bray, A. J., Moore, M. A., J. Phys. C 12 (1979) L 441.

[6] Thouless, D. J., Anderson, P. W., Palmer, R. G., Philos. Mag. 35 (1977) 593. 